Lepr Rev (2000) 71, 123-127

REVIEWS

\title{
The value of DALY life: problems with ethics and validity of disability adjusted life years
}

\author{
TRUDE ARNESEN, ERIK NORD \\ National Institute of Public Health, PB 4404 Torshovw, N-0403 Oslo, \\ Norway
}

Disability adjusted life years (DAL Ys) have been launched by the World Bank and backed by the World Health Organisation as a measure of the global burden of disease. ${ }^{1,2}$ The aim is ambitious: 'The burden of disease has yet to entirely replace traditional approaches to the assessment of health needs as an influence on political decision making. ${ }^{3}$

Just like quality adjusted life years (QALYs), DALYs combine information about morbidity and mortality in numbers of health years lost. In the DALY approach, each state of health is assigned a disability weighting on a scale from zero (perfect health) to one (death) by an expert panel. ${ }^{2}$ To calculate the burden of a certain disease, the disability weighting is multiplied by the number of years lived in that health state and is added to the number of years lost due to that disease (Figure 1). Future burdens are discounted at a rate of $3 \%$ per year, and the value of the lifetime is weighted so that years of life in childhood and old age are counted less.

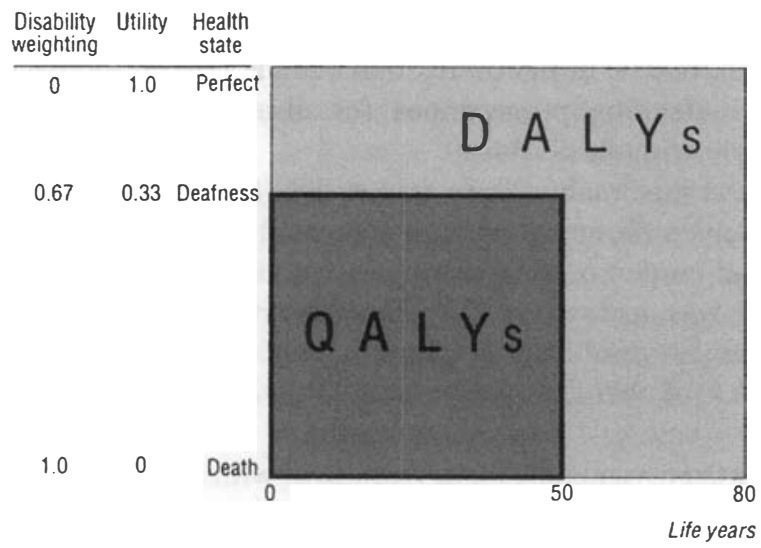

Figure 1. DALYs and QALYs are complementary concepts. QALYs are years of healthy life lived; DALYs are years of healthy life lost. Both approaches multiply the number of years ( $x$ axis) by the quality of those years ( $y$ axis). QALYs use 'utility' weights of health states; DALYs use 'disability weights' to reflect the burden of the same states. For example, if the utility of deafness is 0.67 , the disability weight of deafness is $1-0.67=0.33$. Disregarding age weighting and discounting, and assuming life expectancy of 80 years, a deaf man living 55 years represents $0.67 \times 50=33.4$ QALYs gained and $0.33 \times 50.30 \times 1=46.6$ DALYs lost.

Reproduced with kind permission from BMJ 1999; 319: 1423-1425 


\section{Summary points}

DALYS (disability adjusted life years) have been launched by the World Bank and the World Health Organisation as a combined measure of morbidity and mortality

The DALY approach explicitly presupposes that the lives of disabled people have less value than those of people without disabilities

The method assumes that disabled people are less entitled to scarce health resources for interventions that would extend their lives

These assumptions are in contrast with basic principles of the WHO

Forced consistency between questions that address different issues produces disability weightings that are basically artefacts, this affects the validity of the global burden of disease report

The ongoing revision of the DALY protocol should address these problems

Though the idea of expressing burden of disease in a single index is tempting, any attempt to summarise information about quality of life and length of life in one number is bound to run into conceptual and methodological problems. The DALY review group of the WHO has criticised DALYs for obscuring too much by pressing complex information into a single numeric measure with a mathematical formulation that 'only serves to distract attention from the main issues.' Others have raised objections to the way in which DALYs are currently calculated. ${ }^{4-7}$ Among these objections are that discounting future health gains and losses is disadvantageous for preventive medicine, children, and future generations; that age weighting disfavours children and old people, that the chosen estimates for life expectancy tend to disfavour women; that the expert panels reflect the values of a skewed sample of the population; that age weighting and discounting measures the social usefulness of people's life years rather than the individual utility of life; and that the DALY approach implicitly attaches lower value to life extending programmes for disabled people than to corresponding programmes for people without disability.

Our paper considers this final point in greater detail. After Anand and Hanson noted this problem, ${ }^{5}$ a completely different procedure for weighting disabilities was adopted in the protocol for the global burden of disease project. ${ }^{2}$ In the new procedure, the devaluation of life in disabled people was made explicit. An international research group that intended to use the procedure to establish disability weightings for Europe recently became aware of the offensiveness and lack of validity of the method (see below) and is now finding a new valuation method. ${ }^{8}$

The WHO has started revising the DALY protocol with a view to launching a new version in 2003. This paper aims to draw wider attention to the problems of the existing valuation protocol, in the hope that they be dealt with appropriately when the protocol is revised.

\section{The valuation procedure of the DALY approach}

Valuing health states numerically on a scale of zero to one is the most problematic part of any measure combining quality and quantity of life. The method used in the global burden of 


\section{PTO1 - the first person trade-off question}

You are a decision maker who has enough money to buy only one of two mutually exclusive health interventions. If you purchase intervention A, you will extend the life of 1000 healthy (non-disabled) individuals for exactly one year, at which point they will all die. If you do not purchase intervention $A$, they will all die today. The alternative use of your scarce resources is intervention $B$, with which you can extend the life of $n$ individuals with a particular disabling condition for one year. If you do not buy intervention B they will all die today; if you do purchase intervention B, they will die at the end of exactly one year.

disease project is a specific version of the person trade-off technique, ${ }^{9}$ which was originally developed to include concerns in the process of valuation. ${ }^{10,11}$

In the DALY protocol, expert panels are asked two different person trade-off questions, and consistency between the two answers is then forced. In the first question (PTO1) panellists are asked to compare the value of extended life in people without disabilities with that in disabled people. It is presumed that lifetime of disabled people is worth less than that of people without disabilities and that disabled people have fewer claims on health resources than do people without disabilities. The task is to find out how much less. This is done by means of the question shown in the box. ${ }^{9}$

Deriving a disability weight from PTO1 is described in the summary of the global burden of disease report: ${ }^{12}$ 'if the participant judges that 1000 healthy people would have an equal claim on the resources as 8000 people with some severe disability, the weight assigned to that particular disability is equal to 1 minus 1000 divided by 8000 , or 0.875 .'

In the second person trade-off question (PTO2) subjects are asked to value cures for different chronic conditions relative to interventions that extend life. For instance, how many people cured of blindness does the respondent consider equal to prolonging the lives of 1000 people? If the response is 5000, the corresponding disability weight of blindness is $1000: 5000=0.2$. This question raises the kind of issue that may occur in 'real world' priority setting. Unlike the first question, the second does not presuppose that the lifetime of disabled people is devalued.

\section{The validity of forced consistency}

At this point it might be argued that there is nothing wrong with asking the first question. If people do not want to discriminate between non-disabled and disabled people in matters of life extension, they may simple answer that $n=1000$. Unfortunately, this is not possible since consistency with the other question is forced. To see how this works, consider the case of blindness. Assume that a panellist, on ethical grounds, responds that extending the life of 1000 sighted people and 1000 blind people is equivalent. The resulting disability weighting for blindness is zero. Assume that in the second question the panellist answers that relieving 5000 people of blindness is as valuable as prolonging the lives of 1000 people. This gives a disability weighting of 0.2 . The valuation so far has yielded two different disability weightings for the same health state. The panellist is now asked to reconsider these responses and choose a new pair of answers to produce the same disability weighting. The panellist might end up by selecting PTO1 = 1100 and PT02 $=11,000$, which together yield a disability weighting of 0.09 . This weighting, however, does not correspond to any actual 
preference of the respondent: it is basically an artefact, generated by the requirement for consistency between questions that address different issues.

The disability weightings in use ${ }^{9}$ tell us that the value of one year for 1000 people without disabilities on average is set equivalent to the value of one year for 9524 people with quadriplegia, 4202 people with dementia, 2660 blind people, 1686 people with Down's syndrome without cardiac malformation, 1499 deaf people, 1236 infertile people, and 1025 underweight or overweight people (2 SD from mean weight : height ratio).

\section{Experience of the method}

In May 1998 we participated in a workshop of European researchers working with DALYs. Training sessions to determine the disability weightings of selected illnesses were part of the programme. The sessions followed the global burden of disease protocol, with some adjustments. In PTO1, the response that extended life for 1000 disabled people is as valuable as extended life for 1000 people without disabilities was regarded as unreasonable. Anyone who chose this option was told that he or she was implying that being disabled is as good as being non-disabled and that there is no need to spend resources on disabled people. It was suggested that he or she should therefore indicate a number higher than 1000 .

After the meeting, we sent this summary of our perception of the valuation sessions to the other 11 participants, together with some questions. Eight responded: four agreed with the summary completely, three agreed with most of it, and one disagreed. Seven of the eight said that they thought that the two questions ask about different things and that it should be accepted that they produce different disability weightings. Four subjects felt that they were led 'to some extent' to answer in a particular way, and two subjects felt they were led 'to a great extent'.

In spite of their reported view that the two questions ask about different things, participants at the workshop eventually accepted the requirement of consistency. Some explained that they felt they were participating in a game of little practical consequence. Others reported that rather than making person trade-off judgments, they picked disability weightings that 'looked reasonable' and then selected corresponding person trade-off numbers. Perhaps some also accepted the authority of the facilitators and assumed that they were right in what they were demanding, or tried to avoid unpleasantness.

\section{Confusing value of life with health?}

The line of thought from the first question to the application in cost effectiveness analysis seems to be that the healthier the person, the more valuable their life is to themself and to society and the greater their claim on restricted healthcare resources to have their life extended. This makes sense only if the value of life is not seen as a dimension distinct from health, but rather as a direct positive function of health. In valuing life as a function of health status, the DALY approach is not alone: QALYs have been criticised on the same grounds, ${ }^{13-}$ 18 and often in history people have been classified and dealt with according to their functional capacity.

A valuation of human beings according to their functional capacity is in sharp contrast to the humanistic values laid down in the Declaration of Human Rights: 'recognition of the 
inherent dignity and of the equal and inalienable rights of all members of the human family is the foundation. ${ }^{19}$ The WHO department responsible for the global burden of disease project aims at 'strengthening the scientific and ethical foundations of health policies... The aim of the work is to promote equity, quality, and efficiency. ${ }^{20}$ The current DALY protocol does not seem to accord with this.

\section{Conclusion}

The DALY approach currently in use presupposes that life years of disabled people are worth less than life years of people without disabilities. Through the imposition of consistency between substantially different questions, people participating in evaluation panels are forced to adopt discriminatory positions on the value of life of disabled people. In as much as the disability weightings do not correspond to a clear preference but are the results of forced compromise, they must be seen basically as artefacts. Revision of the DALY protocol should deal with these problems appropriately. In particular, the use of disability weightings in the valuation of gained life years should be abandoned.

1 World Bank. World development report 1993; investing in health. New York: Oxford University Press, 1993.

2 Murray C, Lopez A. The global burden of disease. Cambridge, MA: Harvard University Press, 1996.

3 International Burden of Disease Network. Report of the foundation meeting. Atlanta: International Burden of Disease Network, 1998.

4 Sayers BM, Fliedner TM. The critique of DALYs: a counter-reply. Bull WHO 1997; 75: 383-4.

5 Anand S, Hanson K. Disability adjusted life years: a critical review. J Health Econ 1997; 16: 685-702.

6 Anand S, Hanson K. DALYs: efficiency versus equity. World Development 1998; 26: 307-10.

7 Gwatkin DR. Global burden of disease [letter]. Lancet 1997; 350: 141.

8 Stouthard MEA, Essink-Bot M-L, Bonsel GJ, Barendregt JJ, Kramer PGN, van de Water HPA, et al. Disability weights for diseases in the Netherlands. Rotterdam: Erasmus University, Department of Public Health, 1997.

9 Murray C. Rethinking DALYs. In Murray C, Lopez A, eds. The global burden of disease. Cambridge, MA: Harvard University Press, 1996: 1-98.

10 Patrick DL, Bush JW, Chen M. Methods for measuring levels of well-being for a health status index. Health Serv Res 1978; 8: 228-45.

11 Nord E. Methods for quality adjustment of life years (review). Soc Sci Med 1992; 34: 559-69.

12 Murray C, Lopez A. Summary of the global burden of disease. Cambridge, MA: Harvard University Press, 1996.

13 Harris J. QALYfying the value of life. J Med Ethics 1987; 13: 117-23.

${ }^{14}$ Menzel P. Strong medicine. New York: Oxford University Press, 1990.

15 Harris J. What is the good of health care? Bioethics 1996; 10: 269-91.

16 Nord E, Pinto JL, Richardson J, Menzel P, Ubel P. Incorporating societal concern for fairness in numerical valuations of health programs. Health Econ 1999; 8: 25-39.

17 Savalescu J. Consequentialism, reasons, value and justice. Bioethics 1999; 12: 212-35.

18 Cohen A. On the currency of egalitarian justice. Ethics 1989; 99: 906-44.

19 Universal declaration of human rights. 1948; p.Resolution 217A III UN General Assembly.

20 World Health Organisation. Chester on Evidence and Information for Policy. 98 A.D.; http:/www.who.org/inf-dg/ structure/evidence.html; accessed 30 June 1999. 